\title{
The impact of the Sepsis-3 definition on ICU admission of patients with infection
}

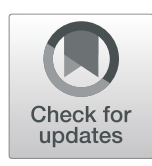

\author{
Jenny Klimpel ${ }^{1}$, Lorenz Weidhase ${ }^{1}$, Michael Bernhard $^{2}$, André Gries $^{3}$ and Sirak Petros ${ }^{1^{*}}$ (D)
}

\begin{abstract}
Background: Sepsis is defined as a life-threatening organ dysfunction due to a dysregulated inflammation following an infection. However, the impact of this definition on patient care is not fully clear. This study investigated the impact of the current definition on ICU admission of patients with infection.
\end{abstract}

Methods: We performed a prospective observational study over twelve months on consecutive patients presented to our emergency department and admitted for infection. We analyzed the predictive values of the quick sequential organ failure assessment (qSOFA) score, the SOFA score and blood lactate regarding ICU admission.

Results: We included 916 patients with the diagnosis of infection. Median age was 74 years (IQR 62-82 years), and 56.3\% were males. There were 219 direct ICU admissions and 697 general ward admissions. A qSOFA score of $\geq 2$ points had $52.9 \%$ sensitivity and $98.3 \%$ specificity regarding sepsis diagnosis. A qSOFA score of $\geq 2$ points had $87.2 \%$ specificity but only $39.9 \%$ sensitivity to predict ICU admission. A SOFA score of $\geq 2$ points had $97.4 \%$ sensitivity, but only $17.1 \%$ specificity to predict ICU admission, while a SOFA score of $\geq 4$ points predicted ICU admission with $82.6 \%$ sensitivity and $71.7 \%$ specificity. The area under the receiver operating curve regarding ICU admission was 0.81 (95 Cl, 0.77-0.86) for SOFA score, 0.55 ( $95 \% \mathrm{Cl}, 0.48-0.61$ ) for blood lactate, and only 0.34 (95\% Cl, 0.28-0.40) for qSOFA on emergency department presentation.

Conclusions: While a positive qSOFA score had a high specificity regarding ICU admission, the low sensitivity of the score among septic patients as well as among ICU admissions considerably limited its value in routine patient management. The SOFA score was the better predictor of ICU admission, while the predictive value of blood lactate was equivocal.

Keywords: Sepsis, qSOFA, SOFA score, Intensive care, Infection, Mortality, Emergency department

\section{Background}

Sepsis is a global medical challenge with a very high incidence and mortality [1-3]. The formulation of a meaningful definition of sepsis remains a challenge, balancing between a clinically prudent definition and that appropriate for research purposes. While the first and second consensus definitions of sepsis were based on the presence of systemic inflammation [4,5], sepsis is currently defined as a life-threatening organ dysfunction due to a dysregulated host response to infection [6-8]. The Sequential Organ Function Assessment (SOFA) Score, which has been in use in intensive care units (ICU) for more than two decades [9], is now at the center of the

\footnotetext{
*Correspondence: sirak.petros@medizin.uni-leipzig.de

${ }^{1}$ Medical ICU, University Hospital of Leipzig, Liebigstr. 20, 04103 Leipzig,

Germany

Full list of author information is available at the end of the article
}

definition of sepsis, and this score is found to be a better prognostic indicator [6].

It is essential to diagnose sepsis at an early stage to positively influence the clinical outcome by initiating appropriate management bundles [10]. The introduction of the quick SOFA (qSOFA) score thus represents the effort to identify high risk patients as early as possible by using basic clinical criteria instead of complex biomarkers [8]. The score includes respiratory rate, Glasgow Coma Scale and systolic blood pressure, based on the analysis preceding the current sepsis definition algorithm. A score of at least two points is considered positive. Several publications have reported the correlation between a positive qSOFA and poor outcome in sepsis patients [6, 11-15]. However, although the qSOFA score represents the shift from the previous notion of systemic

(c) The Author(s). 2019 Open Access This article is distributed under the terms of the Creative Commons Attribution 4.0 International License (http://creativecommons.org/licenses/by/4.0/), which permits unrestricted use, distribution, and 
inflammation now to organ dysfunction as the mainstay of sepsis definition, its impact on the decision-making process is controversial.

Our primary aim was to investigate how the current sepsis criteria influence the decision in the emergency department on the inpatient level of care of patients with infection. Our secondary aim was to assess the impact of the current sepsis criteria on clinical outcome.

\section{Methods}

We conducted a prospective non-interventional observational study at the University Hospital of Leipzig from 01 May 2017 to 30 April 2018. The study was approved by the ethics commission of the University of Leipzig and conducted according to the ethical principles outlined in the Helsinki Accord. For the sake of homogeneity, we have only screened patients with non-surgical infections. Inclusion criterion was the diagnosis of infection based on clinical, laboratory, radiological and/or microbiological evidence, in accordance with published international consensus guidelines for the diagnosis of infections [16-20]. Exclusion criteria were surgical procedure considered a possibility during ward admission, pregnancy, age $<18$ years, refusal to participate in the study, readmission during the study period, and decision for end-of-life care on admission to the emergency department (ED).

We recorded during the ED management demographic data, the presumed or certain focus of infection, the qSOFA score and blood lactate for every patient with presumed infection admitted to either the medical ICU or the general medical wards. Sepsis was defined based on the current Sepsis-3 recommendations as an infection plus at least two new SOFA score points. The SOFA score was computed for patients with the clinical suspicion of sepsis in accordance with the recommendations of the current sepsis definition algorithm [8]. In short, the SOFA score was calculated if in a patient with infection the qSOFA score was positive or, in case qSOFA was negative, if the ED physicians considered sepsis likely. The ED physicians were trained and experienced in applying the algorithms in the management of sepsis. Regarding the SOFA score, the score points presented in this study are not the total score points, but rather the score points considered associated with the current infection. For this purpose, previous electronic patient charts of the hospital as well as information supplied by patients or their guardians or family physicians were evaluated looking for chronic conditions that would influence the SOFA score. In case of missing information, the study group judged the clinical validity of the present variables in relation to the current infection and the SOFA score calculated accordingly.
Additionally, we recorded data on chronic underlying diseases (neurological illnesses requiring home support, chronic pulmonary disease requiring home oxygen therapy or non-invasive ventilation or that resulted in significant limitations in quality of life, chronic heart failure New York Heart Association grade III or IV, liver cirrhosis Child-Pugh class B or C, end-stage renal failure, active hematological malignancy, active solid malignancy and current immunosuppressive therapy).

The medical ICU applies an integration model of intensive care and intermediate care, which allows a flexible use of staff and logistic capacity. The decision for ICU admission was based on published recommendations for intensive care and intermediate care patients $[21,22]$. Thus, the ICU cohort in this study includes both patient subgroups managed by the same team of intensivists and ICU nurses. We analyzed the total ICU cohort as a group, because we believed that further stratification into ICU and intermediate care group would not add any relevant information regarding the main aim of the study. For patients admitted to the ICU, we additionally collected the following data: Acute Physiology And Chronic Health Evaluation (APACHE) II score, the need for vasopressor therapy, invasive mechanical ventilation and renal replacement therapy (RRT), and ICU mortality. We also recorded hospital mortality for the total cohort.

Data were collected daily on an Excel sheet and then transferred to the SPSS sheet after validation by the study group. We performed the statistical analysis using SPSS for Windows version 24 (IBM SPSS, USA). Numerical data are given as either mean with standard deviation or median with interquartile range (IQR) depending on whether they are normally distributed based on Shapiro-Wilk and Kolmogorov-Smirnov tests. We compared numerical data using either the Student $t$ test or the Mann-Whitney U test depending on their normal distribution. Categorical variables were tested using the chi square test with two-sided significance. We calculated the sensitivity, specificity, positive and negative predictive values of the following variables to predict ICU admission: a qSOFA of $\geq 2$ points, a blood lactate of $>2 \mathrm{mmol} / \mathrm{l}$, and a SOFA score of $\geq 2$ and $\geq 4$ points. We constructed receiver operating characteristics (ROC) curves and calculated the area under the ROC (AUROC) regarding ICU admission for positive qSOFA ( $\geq 2$ points) as well as for the SOFA score and blood lactate as continuous variables. A $p$ value $<0.05$ was considered statistically significant.

\section{Results}

We included 916 patients with a median age of 74.0 years (IQR 62-82 years), of whom $56.3 \%$ were males. There were 219 (23.9\%) patients directly admitted to the 
ICU, of whom 166 (75.8\%) fulfilled ICU admission criteria and $53(24.2 \%)$ fulfilled intermediate care criteria. The other $697(76.1 \%)$ patients were admitted to the general wards. Baseline characteristics of the study population are presented in Table 1. Six patients in the ICU admission group and 10 patients in the general ward group with a positive qSOFA score did not fulfill the criteria for sepsis. Chronic lung diseases (15.1\% versus $5.5 \%, p<0.0001)$ and chronic heart failure $(19.7 \%$ versus $9.6 \%, \mathrm{p}<0.0001)$ were significantly more common among ICU admissions than among general ward admissions, while solid malignancies were significantly more common among general ward admissions than among ICU admissions ( $16.5 \%$ versus $8.7 \%, p=0.004)$.

The sensitivity, specificity, positive and negative predictive values of qSOFA, blood lactate and SOFA score in the ED regarding ICU admission are presented in Table 2. The ROC curves of the SOFA score, blood lactate and a positive qSOFA for ICU admission are shown in Fig. 1. The highest AUROC regarding the prediction of ICU admission was demonstrated for SOFA score, while the figure for blood lactate was equivocal and that for qSOFA was low.

Eighteen patients out of the general ward group (2.6\%) had to be transferred to the ICU within $48 \mathrm{~h}$ of admission due to clinical deterioration. Only 5/18 (27.8\%) had a positive qSOFA score on ED admission.

The median SOFA score for patients with sepsis on ED admission was $7.0(4.0-10.5)$ for the ICU admission group and $3.0(2.0-5.0)$ for the general ward group $(p<$ $0.001)$.

Data on disease severity in the ICU for the direct ICU admissions are given in Table 3.

A qSOFA score of $\geq 2$ points had $52.9 \%$ sensitivity and 98.3\% specificity regarding sepsis diagnosis. The hospital mortality rate was $44.7 \%$ among qSOFA-positive ICU admissions, but only $20.3 \%$ among qSOFA-negative ICU admissions $(p<0.0001)$. The hospital mortality in the general ward group was $13.9 \%$ for qSOFA-positive patients, but only $3.7 \%$ for qSOFA-negative patients ( $p=$ 0.001 ). The hospital mortality rate was $38.8 \%$ among lactate-positive (blood lactate $>2.0 \mathrm{mmol} / \mathrm{l}$ ) ICU admissions, but only $17.8 \%$ among lactate-negative (blood lactate $\leq 2.0 \mathrm{mmol} / \mathrm{l})$ ICU admissions $(p=0.001)$. The hospital mortality in the general ward group was $13.2 \%$ for lactate-positive patients, but only $4.1 \%$ for lactatenegative patients $(p<0.0001)$.

\section{Discussion}

This observational study prospectively evaluated the predictive value of the current sepsis definition on ICU admission of patients with infection presenting to the emergency department. The study showed that the SOFA score had a strong correlation with ICU admission. A mere positive SOFA score, defined as at least two score points, used for sepsis definition showed a high sensitivity, but a very low specificity to predict ICU admission in our cohort. A SOFA score of at least four points showed a good sensitivity and specificity to predict ICU admission. This cut-off may help in management decisions in the ED regarding the level of inpatient care. However, this should be independently validated.

In our study, only approximately $40 \%$ of patients directly admitted to the ICU fulfilled the qSOFA criteria. Moskowitz et al. have reported that qSOFA had a high specificity but a poor sensitivity to predict critical care interventions such as vasopressor therapy, assisted ventilation, intravenous fluid substitution, placement of invasive catheters or renal replacement therapy within the first 48 hours [23]. A retrospective study using electronic health records of patients treated for sepsis in the ED reported that qSOFA performed poorly for identifying sepsis in the ED and relying on qSOFA alone may delay initiation of interventions [24]. In a retrospective analysis

Table 1 Baseline characteristics of the study population

\begin{tabular}{|c|c|c|c|c|}
\hline Variable & Total cohort & ICU admission & General ward admission & $p$ \\
\hline $\mathrm{N}(\%$ total) & 916 & $219(23.9)$ & $697(76.1)$ & \\
\hline Male (\%) & 56.3 & 58.4 & 55.7 & 0.48 \\
\hline Median Age (years) with IQR & $74.0(62.0-82.0)$ & $70.0(59.0-78.0)$ & $75.0(62.0-83.0)$ & 0.013 \\
\hline qSOFA $\geq 2$ points (\%) & 23.6 & 39.9 & 12.8 & $<0.0001$ \\
\hline$\geq 1$ chronic disease (\%) & 60.7 & 64.4 & 59.5 & 0.21 \\
\hline Focus of infection (\%) & & & & 0.001 \\
\hline Respiratory & 56.8 & 71.7 & 52.1 & \\
\hline Urogenital & 24.6 & 14.6 & 27.7 & \\
\hline Abdominal & 7.5 & 4.6 & 8.5 & \\
\hline Soft tissue & 5.6 & 3.7 & 6.2 & \\
\hline Others & 5.5 & 5.6 & 5.6 & \\
\hline
\end{tabular}


Table 2 Sensitivity, specificity, positive and negative predictive values of qSOFA, blood lactate and the SOFA score regarding ICU admission

\begin{tabular}{lllll}
\hline Variable & Sensitivity (\%) & Specificity (\%) & Positive predictive value (\%) & Negative predictive value (\%) \\
\hline qSOFA $\geq 2$ points & 39.9 & 87.2 & 49.1 & 82.5 \\
Blood lactate $>$ 2 mmol/l & 58.9 & 58.1 & 31.5 & 81.3 \\
SOFA $\geq 2$ points & 97.3 & 17.1 & 67.6 & 77.8 \\
SOFA $\geq 4$ points & 81.3 & 71.7 & 79.1 & 65.0 \\
\hline
\end{tabular}

of data from more than 30,000 unselected patients in the ED, the sensitivity of qSOFA regarding ICU admission or death was only $53.6 \%$ [25]. The decision to admit septic patients to high level care unit is usually dependent on particular organ dysfunction states. A prospective observational study concluded that clinical judgement is a fast and reliable method to stratify between ICU and general ward admissions in ED patients with sepsis, with qSOFA not adding value to this stratification [26].

The main limitation of qSOFA regarding management decision is its low sensitivity. Szkarmany et al. reported that the risk of missing patients with organ dysfunction while using qSOFA was 30.5\% [27]. Williams et al. have also reported that the poor sensitivity of qSOFA may limit its utility as a bedside screening tool [28]. A metaanalysis showed that the initial qSOFA score is of limited prognostic value in ED patients with infections [29]. A prospective study in patients admitted with infection to the ED reported that qSOFA failed to identify twothirds of patients with severe sepsis [30]. Another retrospective analysis of data including 130,595 adult visits to the ED also concluded that qSOFA is a poor tool for ED sepsis screening [31].

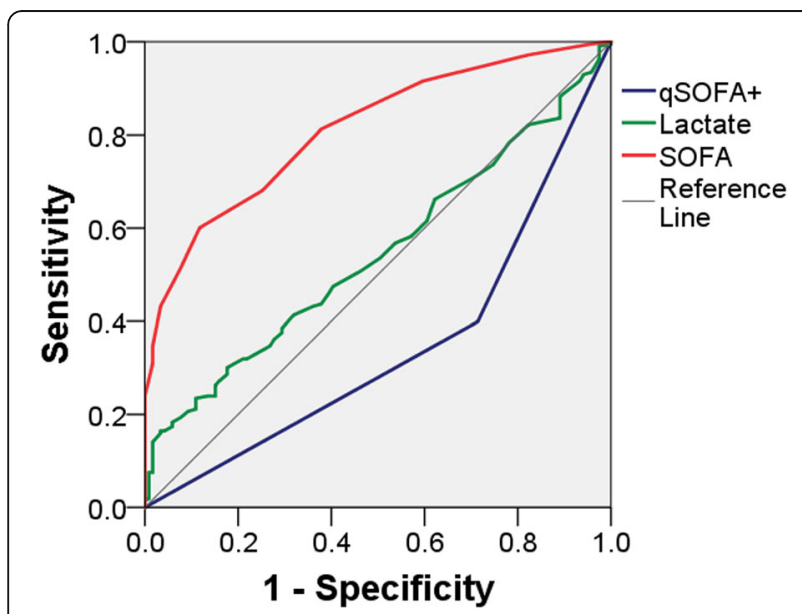

Fig. 1 Receiver operating characteristic curves of the SOFA score, blood lactate and a positive qSOFA for ICU admission based on emergency department admission data. The AUROC was 0.82 (95\% $\mathrm{Cl}, 0.77-0.86)$ for SOFA score, 0.55 (95\% Cl, 0.48-0.61) for blood lactate level, and 0.34 ( $95 \% \mathrm{Cl}, 0.28-0.40)$ for a positive qSOFA
Our study confirmed that patients with a positive qSOFA have a significantly higher hospital mortality rate than those with a negative qSOFA [11, 27, 28, 32-34]. Franchini et al. concluded in their meta-analysis that qSOFA is a higher rule-in than rule-out tool to predict mortality [35].

Our study has certain limitations. Firstly, it was monocentric and non-interventional. However, our data represent the clinical reality in the management of patients with sepsis. Secondly, we have included only non-surgical patients, so that one should be cautious in extrapolating our results to other patient populations. Thirdly, although we have taken every effort to diagnose infection based on available guidelines, we cannot rule out that our cohort might have included patients without any infection. The diagnosis of infection in the first hours of patient presentation remains a challenge. Fourthly, computing the qSOFA score might particularly be challenging in the aging population with a high prevalence of chronic underlying diseases, such as in our cohort. Therefore, these issues should be taken into consideration while comparing our results with those from other studies. Furthermore, we have computed the SOFA score only in those patients with a positive qSOFA and, in case of a negative qSOFA, in those patients with a high clinical suspicion of sepsis, which is the recommended approach by the current definition algorithm [8]. Such a clinical suspicion may be variable depending on the training and experience of emergency physicians. Although we have

Table 3 Disease severity for the ICU admission group during their ICU stay

\begin{tabular}{ll}
\hline Variable & \\
\hline Mean APACHE-II score & $24.7 \pm 7.0$ \\
Mean SOFA score & $7.4 \pm 4.0$ \\
SOFA score $\geq 2$ points (\%) & 97.3 \\
Median Blood lactate (mmol/l) (with IQR) & $1.6(1.1-2.6)$ \\
Blood lactate > 2.0 mmol/I (\%) & 33.3 \\
Invasive ventilation (\%) & 45.7 \\
Vasopressor use (\%) & 53.9 \\
RRT (excluding patients with ESRD) (\%) & 20.1 \\
ICU mortality (\%) & 23.7 \\
Hospital mortality (\%) & 30.0 \\
\hline
\end{tabular}

ESRD end-stage renal disease, IQR interquartile range, $R R T$ renal replacement therapy 
established the definition algorithm in our ED, we cannot rule out that we may have overlooked patients with sepsis. However, the low hospital mortality among our patients managed in the general wards may support our assumption that we could not have overlooked any significant number of sepsis patients. There is no evidence that computing the SOFA score in every admitted patient with infection is meaningful. Calculating the SOFA score may take time while waiting for laboratory results. However, we did not look into this issue and it is also not clear whether this delay might have a significant influence on patient management.

\section{Conclusion}

The SOFA score has a strong impact on ICU admission of patients with infection. An infection-associated SOFA score of at least four points may help in decision making regarding high-level patient care. However, this cut-off should be prospectively validated. Although a positive qSOFA score has a high specificity to diagnosis sepsis and it correlates well with poor outcome, its low sensitivity makes its implementation in the decision-making process questionable.

\section{Acknowledgments}

The authors thank the staff of the emergency department and the medical ICU of the University Hospital of Leipzig for their continuous support.

\begin{abstract}
Authors' contributions
JK designed the study, collected the data, and wrote the manuscript draft. LW designed the study, analyzed the data, and corrected the manuscript draft. MB designed the study, analyzed the data, and corrected the manuscript draft. AG designed the study, collected the data, and corrected the manuscript draft. SP designed the study, analyzed and interpreted the data, and corrected the manuscript draft. All authors have read and approved the final manuscript.
\end{abstract}

\section{Funding}

The study did not receive any funding.

\section{Availability of data and materials}

The datasets during the current study are available from the corresponding author on reasonable request.

\section{Ethics approval and consent to participate}

The study was approved by the ethics commission of the University of Leipzig. Patients were asked to participate in the study after an informed consent.

\section{Consent for publication}

Not applicable.

\section{Competing interests}

The authors declare that they have no competing interests.

\section{Author details}

${ }^{1}$ Medical ICU, University Hospital of Leipzig, Liebigstr. 20, 04103 Leipzig, Germany. ${ }^{2}$ Emergency Department, University Hospital of Düsseldorf, Düsseldorf, Germany. ${ }^{3}$ Emergency Department, University Hospital of Leipzig, Leipzig, Germany.
Received: 23 May 2019 Accepted: 21 October 2019

Published online: 04 November 2019

\section{References}

1. Fleischmann C, Scherag A, Adhikari NK, et al. Assessment of global incidence and mortality of hospital-treated Sepsis. Current estimates and limitations. Am J Respir Crit Care Med. 2016:193:259-72.

2. Esteban A, Frutos-Vivar F, Ferguson ND, et al. Sepsis incidence and outcome: contrasting the intensive care unit with the hospital ward. Crit Care Med. 2007;35:1284-9.

3. Gaieski DF, Edwards JM, Kallan MJ, Carr BG. Benchmarking the incidence and mortality of severe sepsis in the United States. Crit Care Med. 2013:41:1167-74.

4. Bone RC, Balk RA, Cerra FB, et al. American College of Chest Physicians/ Society of Critical Care Medicine consensus conference: definitions for sepsis and organ failure and guidelines for the use of innovative therapies in sepsis. Crit Care Med. 1992;20:864-74.

5. Levy MM, Fink MP, Marshall JC, et al. 2001 SCCM/ESICM/ACCP/ATS/SIS international Sepsis definitions conference. Intensive Care Med. 2003; 29:530-8.

6. Seymour CW, Liu VX, Iwashyna TJ, et al. Assessment of clinical criteria for Sepsis: for the third international consensus definitions for Sepsis and septic shock (Sepsis-3). JAMA. 2016;315:762-74.

7. Shankar-Hari M, Phillips GS, Levy ML, et al. Developing a new definition and assessing new clinical criteria for septic shock: for the third international consensus definitions for Sepsis and septic shock (Sepsis3). JAMA. 2016;315:775-87.

8. Singer $M$, Deutschman $C S$, Seymour $C W$, et al. The third international consensus definitions for Sepsis and septic shock (Sepsis-3). JAMA. 2016;315:801-10

9. Vincent JL, Moreno R, Takala J, et al. The SOFA (Sepsis-related organ failure assessment) score to describe organ dysfunction/failure. On behalf of the working group on Sepsis-related problems of the European Society of Intensive Care Medicine. Intensive Care Med. 1996;22:707-10.

10. Rhodes A, Evans LE, Alhazzani W, et al. Surviving Sepsis campaign: international guidelines for Management of Sepsis and Septic Shock: 2016. Intensive Care Med. 2017:43:304-77.

11. Freund $Y$, Lemachatti $N$, Krastinova E, et al. Prognostic accuracy of Sepsis-3 criteria for in-hospital mortality among patients with suspected infection presenting to the emergency department. JAMA. 2017;317:301-8.

12. Singer AJ, Ng J, Thode HC Jr, Spiegel R, Weingart S. Quick SOFA Scores predict mortality in adult emergency department patients with and without suspected infection. Ann Emerg Med. 2017;69:475-9.

13. Canet E, Taylor DM, Khor R, Krishnan V, Bellomo R. qSOFA as predictor of mortality and prolonged ICU admission in emergency department patients with suspected infection. J Crit Care. 2018;48:118-23.

14. Jiang J, Yang J, Jin Y, Cao J, Lu Y. Role of qSOFA in predicting mortality of pneumonia: a systematic review and meta-analysis. Medicine (Baltimore). 2018;97:e12634.

15. Gaini S, Relster MM, Pedersen C, Johansen IS. Prediction of 28-days mortality with sequential organ failure assessment (SOFA), quick SOFA (qSOFA) and systemic inflammatory response syndrome (SIRS) - a retrospective study of medical patients with acute infectious disease. Int J Infect Dis. 2019;78:1-7.

16. Calandra T, Cohen J. The international sepsis forum consensus conference on definitions of infection in the intensive care unit. Crit Care Med. 2005:33:1538-48.

17. Mandell LA, Wunderink RG, Anzueto A, et al. Infectious Diseases Society of America/American Thoracic Society consensus quidelines on the management of community-acquired pneumonia in adults. Clin Infect Dis. 2007:44(Suppl 2):S27-72.

18. Woodhead M, Blasi F, Ewig S, et al. Guidelines for the management of adult lower respiratory tract infections--full version. Clin Microbiol Infect. 2011; 17(Suppl 6):E1-59.

19. Solomkin JS, Mazuski JE, Bradley JS, et al. Diagnosis and management of complicated intra-abdominal infection in adults and children: guidelines by the surgical infection society and the Infectious Diseases Society of America. Clin Infect Dis. 2010;50:133-64.

20. Stevens DL, Bisno AL, Chambers HF, et al. Practice guidelines for the diagnosis and management of skin and soft tissue infections: 2014 update by the Infectious Diseases Society of America. Clin Infect Dis. 2014;59:e10-52. 
21. Nates JL, Nunnally M, Kleinpell R, et al. ICU admission, discharge, and triage guidelines: a framework to enhance clinical operations, development of institutional policies, and further research. Crit Care Med. 2016;44:1553-602.

22. Nasraway SA, Cohen IL, Dennis RC, et al. Guidelines on admission and discharge for adult intermediate care units. American College of Critical Care Medicine of the Society of Critical Care Medicine. Crit Care Med. 1998; 26:607-10.

23. Moskowitz A, Patel PV, Grossestreuer AV, et al. Quick sequential organ failure assessment and systemic inflammatory response syndrome criteria as predictors of critical care intervention among patients with suspected infection. Crit Care Med. 2017:45:1813-9.

24. Haydar S, Spanier M, Weems P, Wood S, Strout T. Comparison of QSOFA score and SIRS criteria as screening mechanisms for emergency department sepsis. Am J Emerg Med. 2017;35:1730-3.

25. Churpek MM, Snyder A, Han X, et al. Quick Sepsis-related organ failure assessment, systemic inflammatory response syndrome, and early warning scores for detecting clinical deterioration in infected patients outside the intensive care unit. Am J Respir Crit Care Med. 2017;195:906-11.

26. Quinten VM, van Meurs M, Wolffensperger AE, Ter Maaten JC, Ligtenberg $J \mathrm{JM}$. Sepsis patients in the emergency department: stratification using the clinical impression score, predisposition, infection, response and organ dysfunction score or quick sequential organ failure assessment score? Eur J Emerg Med. 2018;25:328-34.

27. Szakmany T, Pugh R, Kopczynska M, et al. Defining sepsis on the wards: results of a multi-Centre point-prevalence study comparing two sepsis definitions. Anaesthesia. 2018;73:195-204.

28. Williams JM, Greenslade JH, McKenzie JV, Chu K, Brown AFT, Lipman J. Systemic inflammatory response syndrome, quick sequential organ function assessment, and organ dysfunction: insights from a prospective database of ED patients with infection. Chest. 2017;151:586-96.

29. Jiang J, Yang J, Mei J, Jin Y, Lu Y. Head-to-head comparison of qSOFA and SIRS criteria in predicting the mortality of infected patients in the emergency department: a meta-analysis. Scand J Trauma Resusc Emerg Med. 2018;26:56.

30. Askim A, Moser F, Gustad LT, et al. Poor performance of quick-SOFA (qSOFA) score in predicting severe sepsis and mortality - a prospective study of patients admitted with infection to the emergency department. Scand J Trauma Resusc Emerg Med. 2017;25:56.

31. Usman OA, Usman AA, Ward MA. Comparison of SIRS, QSOFA, and NEWS for the early identification of sepsis in the emergency department. Am J Emerg Med. 2019;37:1490-7.

32. Giamarellos-Bourboulis EJ, Tsaganos T, Tsangaris I, et al. Validation of the new Sepsis-3 definitions: proposal for improvement in early risk identification. Clin Microbiol Infect. 2017;23:104-9.

33. April MD, Aguirre J, Tannenbaum LI, et al. Sepsis clinical criteria in emergency department patients admitted to an intensive care unit: an external validation study of quick sequential organ failure assessment. J Emerg Med. 2017;52:622-31.

34. Brink A, Alsma J, Verdonschot R, et al. Predicting mortality in patients with suspected sepsis at the emergency department; a retrospective cohort study comparing qSOFA, SIRS and National Early Warning Score. PloS one. 2019;14:e0211133.

35. Franchini S, Scarallo L, Carlucci M, Cabrini L, Tresoldi M. SIRS or qSOFA? Is that the question? Clinical and methodological observations from a metaanalysis and critical review on the prognostication of patients with suspected sepsis outside the ICU. Intern Emerg Med. 2019;14:593-602.

\section{Publisher's Note}

Springer Nature remains neutral with regard to jurisdictional claims in published maps and institutional affiliations.

Ready to submit your research? Choose BMC and benefit from:

- fast, convenient online submission

- thorough peer review by experienced researchers in your field

- rapid publication on acceptance

- support for research data, including large and complex data types

- gold Open Access which fosters wider collaboration and increased citations

- maximum visibility for your research: over $100 \mathrm{M}$ website views per year

At BMC, research is always in progress.

Learn more biomedcentral.com/submissions 